\title{
Convective Instability in Binary Nanofluids for Absorption Phenomenon with Cross Diffusions and Internal Heat Source
}

\author{
Sravan N. Gaikwad*, Dnyaneshwar M. Surwase \\ Department of Mathematics, Gulbarga University, Kalaburagi 585 106, India
}

Corresponding Author Email: sarvangaikwad@gug.ac.in

https://doi.org/10.18280/ijht.390402

Received: 29 December 2020

Accepted: 2 August 2021

\section{Keywords:}

binary nanofluid, convection, cross diffusions, Dufour effect, heat generation, separation ratio, thermophoresis

\begin{abstract}
Theoretical investigation of cross diffusion effects on the convective stability of binary nanofluids under the influence of internal heat source is carried out using linear stability theory and addition factor analysis. Cross diffusions include Soret and Dufour effects. Soret effect implies that mass diffusion induced by thermal gradient and Dufour effect implies that heat transfer induced by concentration gradient. The physical system containing a binary nanofluid of rigid, impermeable to mass flow and perfectly thermal conducting boundaries where temperatures and concentrations are constant but different is taken. The stability equations are framed and the formulation of Rayleigh numbers is made for stationary and oscillatory states of the system. The effects of dimensionless parameters like internal Rayleigh number, Soret coefficients of nanoparticles and solute, volume fraction of nanoparticles and Dufour coefficient on the system are represented graphically. Ammonia/water based silver and alumina, the two binary nanofluids are considered and impact of nanoparticles through the addition factor on stability of the system is discussed pictorially. It is observed that the internal Rayleigh number reduces the size of convection cells. It causes to destabilize the system and improves the heat and concentration transfer in the system. Stability of the system under the influence of cross diffusion depends upoun the Soret effects of solute and nanoparticles. The Soret parameter and volume fraction of nanoparticles enlarge the size of convection cells and Dufour parameter reduces the size of convection cells in stationary state of the system. The reversed effects are reported in case of oscillatory state. The volume fraction of nanoparticles has destabilizing effect and it causes to increase the heat transfer in binary nanofluid.
\end{abstract}

\section{INTRODUCTION}

Nanofluid, a fluid prepared by disseminating the nanometer-sized particles of metals, metallic oxides, carbides or carbon nanotubes steadily and consistently in regular host fluids like ethylene glycol, oil, water, etc. For the past decades, it plays a vital role in many fields owing to its extremely intensify thermal properties and significant applications as heat transfer fluids, ferromagnetic fluids, superwetting fluids and detergents, biomedical fluids, polymer nanocomposites, gain media in random lasers, building blocks for electronics and optoelectronics devices, etc. [1,2]. Choi and Eastman [3] were the first, who proposed the term 'nanofluid'. Many industrial processes like air conditioning and refrigeration, automobiles, distillation of solar water, foods freezing, etc. [46] are based on absorption phenomenon. In these processes, the binary mixture of fluids such as Ammonia - Ethelyne glycol, Ammonia - Sodium bromide, Ammonia - Water, Lithium bromide - Water, Carbon dioxide - Water, etc. are used instead of single fluids as host fluids. The binary nanofluids are the nanofluids in which the host fluids are such binary mixtures [7]. The experimental observation on the enhancement of heat and mass transfer in binary nanofluids for absorption processes has been done by Ma et al. [8], Lee et al. [9] and Pang et al. [10]. They have shown that the effect of shape and size of nanoparticles is more significant for enhancement of heat and mass transfer in binary nanofluids. It is studied by Xuan [11] theoretically and reported that the micro motion and stirring of suspended nanoparticles enhance the mass transport process in binary nanofluids. Such enhancement of mass diffusion in binary nanofluids depends on the size, volume fraction and state of motion of nanoparticles.

The convective instability in nanofluids has recently received considerable attention due to its numerous applications in many engineering phenomena such as chemical engineering, geothermal energy utilization, oil reservoir modeling, building of thermal insulation, solar energy, lubrication and biological processes $[12,13]$. The convective stability problems in binary nanofluids using Buongiorno's transport model [14] have been studied by many researchers for various situations. Few of the examinations are Nield and Kuznetsov [15], Yadav et al. [16], Bhadauria et al. [17] and Gupta et al. [18]. The study on convective instability driven by buoyancy have been carried out by Kim et al. [19] by using an addition factor analysis and reported that the effect of addition of nanoparticles to the basefluid is to set in convective motion easily in nanofluid layer. Further, the convective stability in binary nanofluids for absorption phenomena with thermodiffusion and diffusiothermal effect of nanoparticles is studied theoretically by Kim et al. [20, 21]. They have shown that both effects decrease the stability of system of nanofluid and increase the transfer of heat more significantly.

Since few decades, the convective heat and mass transfer 
due to internal heating has got much attention because of its wide spread applications in the engineering problems related to energy like nuclear reactions, nuclear heat cores, nuclear energy, nuclear disposals, oil extractions, solar collectors, crystal growth, geophysical systems, etc. [22, 23]. The common phenomena of convections in nature induced by internal heat source include a motion in the atmosphere where heat is generated by absorption of sunlight and mantle convection in the earth where heat is generated by the radioactive decay of isotopes. Internal heating is the main source of convective heat and mass transfer caused by temperature gradient of the system. It keeps the system warm and active. Yadav et al. [24] were the first, who analysed the effect of internal heat source on stability of nanofluid layer with Brownian diffusion and thermophoresis of nanoparticles. It has been reported that the internal heat generating nanofluid layer increases the onset of convection in the system. The convection in an internally heated nanofluid layer considering the different boundaries as free-free, rigid-free and rigid-rigid is studied by Nield and Kuznetsov [25], and shown that the combinations of modified diffusivity ratio with nanoparticle Rayleigh number and Lewis number lead to make the nanofluid layer more unstable. Very recently, the effect of internal heating on stability of Rayleigh-Benard convection in different water-based nanoliquids and heat transfer enhancement of the nanoliquids through different shapes of enclosures has analysed by Kanchana and Zhao [26]. Gaikwad and Surwase [27] have studied the convection phenomena in binary nanofluids with Soret effects and internal heat generation.

Keeping in view of the literature cited above, it seems that the problems of convective stability of binary nanofluids are of more interesting in the fields based on absorption phenomenon. However, theoretical work on this is rare. So, this motivated to do the present study with cross diffusion effects by carrying out addition factor analysis under the influence of internal heat source.

\section{PROBLEM FORMULATION}

A horizontal layer of incompressible binary nanofluid which is at rest and lying in the region $0 \leq z \leq h$ is considered as shown in the Figure 1. The $\mathrm{x}$-axis and $\mathrm{z}$-axis are taken along horizontal and vertical directions respectively. The acceleration due to gravity $\bar{g}$ is acting in the negative direction of z-axis. The boundaries of the layer are taken to be rigid, impermeable to mass flow and perfectly thermal conducting where temperatures and concentrations are constant but different. At the lower boundary $z=0$, temperatures and concentrations are $T_{0}+\Delta T$ and $C_{0}+\Delta C$ respectively and at the upper boundary $z=h$, temperatures and concentrations are $T_{0}$ and $C_{0}$ respectively. When the binary nanofluid layer is heated from below, the buoyancy driven convection (Rayleigh Benard convection) caused by temperature and concentration difference sets in over a certain thermal gradient.

\subsection{Governing equations}

The governing equations employing the Boussinesq approximations to study the convection in binary nanofluid under the influence of internal heat source with cross diffusions in dimensional form [21, 24, 27] are:

$$
\begin{gathered}
\nabla \cdot \bar{q}=0 \\
\rho_{0}\left(\frac{\partial}{\partial t}+\bar{q} \cdot \nabla\right) \bar{q}=-\nabla P+\mu \nabla^{2} \bar{q}+\rho \bar{g} \\
\left(\frac{\partial}{\partial t}+\bar{q} \cdot \nabla\right) T=\alpha \nabla^{2} T+D_{\mathrm{df}} \nabla^{2} C+Q\left(T-T_{0}\right) \\
\left(\frac{\partial}{\partial t}+\bar{q} \cdot \nabla\right) C=D \nabla^{2} C+D_{\mathrm{sr}} \nabla^{2} T \\
\rho=\rho_{0}\left(1-\beta_{T}\left(T-T_{0}\right)+\beta_{S}\left(C-C_{0}\right)\right)
\end{gathered}
$$

The physical parameters involved in the Eqns. (1)-(5) have their typical sense and are prescribed in the nomenclature.

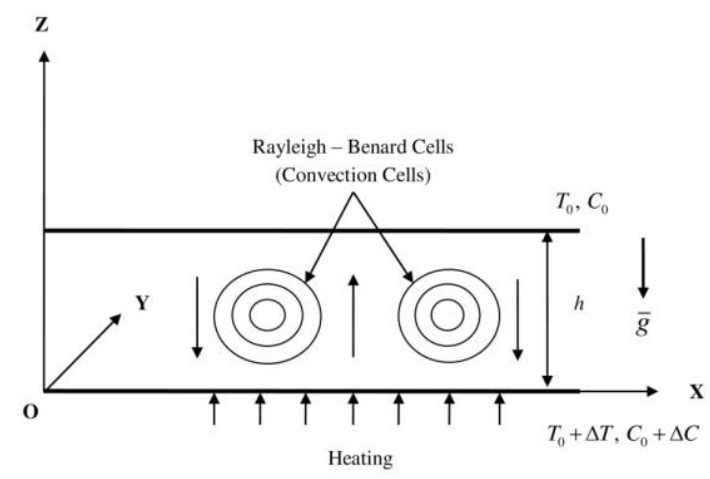

Figure 1. Geometrical configuration of physical system

\subsection{Conduction state analysis}

Employing the following time independent forms of the quantities: $\quad \bar{q}=\bar{q}_{b}=(u, v, w)=(0,0,0), P=P_{b}(z), T=$ $T_{b}(z), C=C_{b}(z), \rho=\rho_{b}(z)$ and $\bar{g}=(0,0,-g)$ in Eqns. (1)-(5), the temperature and concentration at the basic state are given by:

$$
\begin{gathered}
T_{b}=T_{0}+\Delta T\left(\frac{\sin \left(\sqrt{\frac{R i}{1-\kappa}}\left(1-\frac{z}{h}\right)\right)}{\sin \left(\sqrt{\frac{R i}{1-\kappa}}\right)}\right) \\
C_{b}=C_{0}+\Delta C\left(1-\frac{z}{h}\right)
\end{gathered}
$$

where, $\kappa=\frac{D_{\mathrm{sr}} D_{\mathrm{df}}}{\alpha D}$, and $R i=\frac{Q h^{2}}{\alpha}$ is internal Rayleigh number.

\section{LINEAR STABILITY ANALYSIS}

\subsection{Perturbed state analysis}

The infinitesimal disturbances are superposed on the 
quantities in conduction state:

$\bar{q}=\bar{q}_{b}+q^{\prime}, P=P_{b}+P^{\prime}, T=T_{b}+T^{\prime}, C=C_{b}+C^{\prime}, \rho=\rho_{b}+\rho^{\prime}$,

the following equations are obtained:

$$
\nabla \cdot q^{\prime}=0
$$

$$
\begin{aligned}
&\left(\frac{\partial}{\partial t^{\prime}}+q^{\prime} \cdot \nabla\right) q^{\prime}=-\frac{1}{\rho_{0}} \nabla P^{\prime}+ \\
&+\left(\beta_{S} C^{\prime}-\beta_{T^{\prime}} T^{\prime}\right) \bar{g}+v \nabla^{2} q^{\prime} \\
&\left(\frac{\partial}{\partial t^{\prime}}+q^{\prime} \cdot \nabla\right) T^{\prime}+\left(\frac{\Delta T}{h} f(z)\right) w^{\prime}= \\
&\left(\frac{\partial}{\partial t^{\prime}}+q^{\prime} \cdot \nabla\right) C^{\prime}-\left(\frac{\beta_{T} C_{s, i} \Delta T}{h}\right) w^{\prime}=D \nabla^{2} C^{\prime}+D_{\mathrm{df}} \nabla^{2} C+Q T^{\prime} \\
&+D_{\mathrm{sr}} \nabla^{2} T^{\prime}
\end{aligned}
$$

where, $q^{\prime}, P^{\prime}, T^{\prime}$ and $C^{\prime}$ are the perturbed quantities.

Linearizing the Eqns. (9)-(11), introducing the nondimensional variables $(x, y, z)=\frac{1}{h}\left(x^{\prime}, y^{\prime}, z^{\prime}\right), t=\frac{\alpha}{h^{2}} t^{\prime}, \bar{q}=\frac{h}{\alpha} q^{\prime}, T=\frac{T^{\prime}}{\Delta T}, C=\frac{C^{\prime}}{\Delta C}$ in the Eqns. (8)-(11) and then eliminating the pressure gradient term from reduced equation of Eq. (9), the following nondimensional equations are obtained:

$$
\begin{gathered}
\left(\frac{1}{\operatorname{Pr}} \frac{\partial}{\partial t}-\nabla^{2}\right) \nabla^{2} w=\left(\frac{\beta_{s} \Delta C h^{3} g}{\alpha v}\right) \nabla_{1}^{2} C \\
-R a \nabla_{1}^{2} T \\
\left(\frac{\partial}{\partial t}-\nabla^{2}-R i\right) T=f(z) w+\left(\frac{D_{\mathrm{df}} \Delta C}{\alpha \Delta T}\right) \nabla^{2} C \\
\left(\frac{\partial}{\partial t}-\frac{1}{L e} \nabla^{2}\right) C=\left(\frac{\beta_{T} C_{s, i} \Delta T}{\Delta C}\right) w \\
+\left(\frac{D_{\mathrm{sr}} \Delta T}{\alpha \Delta C}\right) \nabla^{2} T
\end{gathered}
$$

subjected to the boundary conditions:

$$
\begin{aligned}
& w=0, \frac{\partial w}{\partial z}=0, T=0, C=0 \text { at } z=0 \\
& w=0, \frac{\partial w}{\partial z}=0, T=0, C=0 \text { at } z=h
\end{aligned}
$$

where, $f(z)=\frac{\partial T_{b}}{\partial z} \quad$ is temperature gradient, $R a=\frac{\beta_{T} \Delta T g h^{3}}{\alpha v}$ is thermal Rayleigh number, $\operatorname{Pr}=\frac{v}{\alpha}$ is Prandtl number, $L e=\frac{\alpha}{D}$ is Lewis number.

\subsection{Stability equations}

The disturbances in $w, T$ and $C$ are assumed to show the level of periodicity by the normal mode technique [28]:

$$
\begin{aligned}
& w(x, y, z, t)=W(z) \exp (i(l x+m y)+\sigma t) \\
& T(x, y, z, t)=\Theta(z) \exp (i(l x+m y)+\sigma t) \\
& C(x, y, z, t)=\Phi(z) \exp (i(l x+m y)+\sigma t)
\end{aligned}
$$

where, $\sigma$ is the growth rate of disturbances. Introducing Eq.(16) into Eqns. (12)-(14), the following stability equations are obtained:

$$
\begin{gathered}
\left(\mathrm{D}^{2}-a^{2}\right)\left(\left(\mathrm{D}^{2}-a^{2}\right)-\frac{1}{\mathrm{Pr}} \sigma\right) W \\
+\left(a^{2} R a\right) \Theta-a^{2}\left(\frac{\beta_{s} \Delta C h^{3} g}{\alpha \nu}\right) \Phi=0 \\
\left(\left(\mathrm{D}^{2}-a^{2}\right)-\sigma+R i\right) \Theta \\
+\frac{D_{\mathrm{df}} \Delta C}{\alpha \Delta T}\left(\mathrm{D}^{2}-a^{2}\right) \Phi+f(z) W=0 \\
\left(\mathrm{D}^{2}-a^{2}\right) \Theta+\left(\frac{D \Delta C}{D_{\mathrm{sr}} \Delta T}\left(\mathrm{D}^{2}-a^{2}\right)-\frac{\alpha \Delta C}{D_{\mathrm{sr}} \Delta T} \sigma\right) \Phi \\
-\left(\frac{\beta_{T} \alpha C_{s, i}}{D_{\mathrm{sr}}}\right) W=0
\end{gathered}
$$

At fundamental mode, $W=\sin (\pi z)$, is assumed to be the most unstable solution of Eqns. (17)-(19). The important parameter to analyse the convective instability under the influence of internal heating in binary nanofluids, the thermal Rayleigh number $R a$ is determined as:

$$
R a=\frac{\delta^{2}\left(\delta^{2}+\frac{\sigma}{\operatorname{Pr}}\right)\left(\delta^{4}(1-\kappa)+\delta^{2}(\sigma+R i)+L e \sigma\left(\delta^{2}+\sigma+R i\right)\right)}{a^{2}\left(2 F\left(\delta^{2}+L e \sigma+\delta^{2} S_{r}\right)-\delta^{2} D_{u}-S_{o}\left(\delta^{2}+\sigma+R i\right)\right)}
$$

where, $\quad \delta^{2}=\pi^{2}+a^{2} \quad$ and $\quad F=\int_{0}^{1} f(z) \sin ^{2}(\pi z) d z \quad$, $S_{o}=\frac{\beta_{s} C_{s, i} \alpha}{D}$ is solutal parameter, $S_{r}=\frac{\beta_{s} D_{\mathrm{sr}}}{\beta_{T} D}$ is the Soret parameter and $D_{u}=\frac{\beta_{T} C_{s, i} D_{\mathrm{df}}}{D}$ is the Dufour parameter. 


\subsection{Stationary convection}

At the marginally stable steady state $\left(\sigma=\omega_{r}+i \omega_{i}=0\right)$, for the reliability of exchange of stability [29, 30], the Eq. (20) can be written as:

$$
R a^{s t}=\frac{\delta^{6}\left(\delta^{2}(1-\kappa)+R i\right)}{a^{2}\left(\delta^{2}\left(2 F\left(1+S_{r}\right)-D_{u}\right)-S_{o}\left(\delta^{2}-R i\right)\right)}
$$

Without considering the influence of internal heating (i.e. for $R i=0$ and $F=-1 / 2$ ), the Eq. (21) reduces to:

$$
R a^{s t}=\frac{\delta^{6}(1-\kappa)}{a^{2}\left(1+S_{o}+S_{r}+D_{u}\right)}
$$

which is the result given by Kim et al. [21] for stationary convection in binary nanofluids.

\subsection{Oscillatory convection}

The thermal Rayleigh number for oscillatory convection by setting $\sigma=i \omega_{i}$ in Eq. (20), is obtained as:

$$
R a=\Delta_{1}+i \omega_{i} \Delta_{2}
$$

The expressions of $\Delta_{1}$ and $\Delta_{2}$ are provided in the Appendix. The Rayleigh number $R a$ is to be real because it is a physical quantity. So, from Eq. (23), either $\omega_{i}=0$ or $\Delta_{2}=0$. But $\omega_{i} \neq 0$ for the onset of oscillatory convection. Thus, the formulation for oscillatory frequency $\omega$ is given by taking $\Delta_{2}=0$ (dropping subscript $i$ ):

$$
\omega^{2}=\frac{A_{1} A_{3}-A_{2} A_{6}}{A_{1} A_{4}-A_{2} A_{5}}
$$

Now Eq. (23) with $\Delta_{2}=0$ gives

$$
R a^{o s c}=\frac{B_{1} \omega^{4}+B_{2} \omega^{2}+B_{3}}{B_{4} \omega^{2}+B_{5}}
$$

The parameters $A_{1}, A_{2}, A_{3}, A_{4}, A_{5}, A_{6}, B_{1}, B_{2}, B_{3}, B_{4}$, and $B_{5}$ are given in the Appendix. In order to investigate the effects of various physical parameters on the onset of oscillatory convection, the Eq. (25) is minimized with the help of Eq. (24).

\section{IMPACT OF NANOPARTICLES}

\subsection{Mixture theory in binary nanofluids and phenomenological models}

The relations for density and heat capacity of binary nanofluid at thermal equilibrium state according to mass conservation law are respectively given by

$$
\rho_{n f}=\left(1-\phi_{v}\right) \rho_{b f}+\phi_{v} \rho_{n p}
$$

and

$$
\left(\rho C_{p}\right)_{n f}=\left(1-\phi_{v}\right)\left(\rho C_{p}\right)_{b f}+\phi_{v}\left(\rho C_{p}\right)_{n p}
$$

The Brinkman model for viscosity and Bruggeman model for thermal conductivity [19] of binary nanofluids are respectively:

$$
\mu_{n f}=\frac{\mu_{b f}}{\left(1-\phi_{v}\right)^{2.5}}
$$

and

$$
k_{n f}=\frac{\left(k_{B}+\sqrt{\left(k_{B}\right)^{2}+8 \delta_{0}}\right) k_{b f}}{4}
$$

where, $k_{B}=\left(3 V_{e} \phi_{v}-1\right) \delta_{0}+\left(3\left(1-V_{e} \phi_{v}\right)-1\right)$ and $\delta_{0}=\frac{k_{n p}}{k_{b f}}$ is the ratio of thermal conductivity of nanoparticles to that of basefluid.

\subsection{Addition factor analysis}

It is familiar that in binary nanofluids, the concentration of solute and nanoparticles are:

$$
C_{s}=\left(\frac{1-\phi_{\mathrm{v}}}{1-\phi_{\mathrm{v}}\left(1-\delta_{1}\right)}\right) C
$$

and

$$
C_{n p}=\left(\frac{\phi_{\mathrm{v}} \delta_{1}}{1-\phi_{\mathrm{v}}\left(1-\delta_{1}\right)}\right) C
$$

where, $\delta_{1}=\frac{\rho_{n p}}{\rho_{b f}}$ is the ratio of densities. The Eq. (27), can be written as:

$$
\frac{\left(\rho C_{p}\right)_{n f}}{\left(\rho C_{p}\right)_{b f}}=1-\phi_{v}\left(1-\delta_{2}\right)
$$

where, $\delta_{2}=\frac{\left(\rho C_{p}\right)_{n p}}{\left(\rho C_{p}\right)_{b f}}$ is the ratio of heat capacities in binary nanofluids. Incorporating the Eqns. (30) and (31) into Eq. (4), the separation ratio $\psi$ is obtained as:

$$
\psi=\left(\frac{1-\phi_{v} \delta_{1}\left(1-\delta_{4}\right)}{1-\phi_{v} \delta_{1}\left(1-\delta_{3}\right)}\right) \psi_{b f}
$$

where, $\psi_{b f}$ is separation ratio in solute (also called as Soret effect of solute), $\delta_{3}=\frac{D_{n p}}{D_{S}}$ and $\delta_{4}=\frac{(D S)_{n p}}{(D S)_{s}}$ are the ratio of diffusivities and the ratio of the Soret coefficients in binary 
nanofluids respectively. The separation ratio $\psi$ contains the Soret effect of nanoparticles $\delta_{4}$ and Soret effect of solute $\psi_{b f}$. Thus, it plays a role analogous to the Soret parameter $S_{r}$ in the binary mixtures. Using above procedure, the correlation for Rayleigh numbers [19] is written as:

$$
R a_{n f}=F_{a} R a_{b f}
$$

where, $F_{a}$ is addition factor which performs a crucial role to examine the impact of nanoparticles on convective stability of binary nanofluids.

\section{RESULTS AND DISCUSSION}

The convective instability of binary nanofluids in the presence of cross diffusions and internal heat source is carried out with the help of linear stability theory and addition factor analysis. The linear stability theory is used to extend the understanding of flow dynamics observed and it is based on linear disturbance equations (stability equations). The addition factor analysis is an analysis done by introducing an addition factor in the correlation of Rayleigh numbers of binary nanofluid and base fluid. Rayleigh numbers which characterize the stability of the system are derived analytically for stationary and oscillatory convections using linear stability theory. The effect of nanoparticles on stability of the system is analysed using addition factor $F_{a}$. The thermophysical properties of basefluids and nanoparticles used are given in Table 1. For various combinations of internal Rayleigh number $R i$ and volume fraction $\phi_{v}$, the Rayleigh number $R a_{c}^{s t}$ is computed to validate the results obtained and listed in Table 2. The obtained results are matched with previous published result [21] for $R i=0$. The parameters used in the present study, solute diffusivity $(D)$, thermal expansion coefficient $\left(\beta_{T}\right)$ heat source parameter $(R i)$, volume fraction $\left(\phi_{v}\right)$, Lewis number $(L e)$, Soret effect of nanoparticle $\left(\delta_{4}\right)$ and separation ratio in binary basefluid $\left(\psi_{b f}\right)$ are taken in range of $10^{-2}-10^{-6}, 10^{-1}-10$ ${ }^{3}, 0-200,0-1,0-10,-5-5$, and $-50-50$, respectively [20, 24, 31, 32].

Figure 2 illustrates the impact of internal Rayleigh number $R i$ on temperature distribution $T_{b}(z)$ at conduction state. The profiles of the temperature distribution move upward with an increase of internal Rayleigh number $R i$, which in turn $R i$ increases the strength of the temperature distribution in positive direction and improves the disturbance in the layers of binary nanofluid. It means that the internal Rayleigh number $R i$ makes the binary nanofluid layer more unstable.

Table 1. Physical parameters of host fluids (Water/Ammonia) and nanoparticles [31, 32]

\begin{tabular}{ccccc}
\hline \multirow{2}{*}{ Physical parameters } & \multicolumn{2}{c}{ Base Fluids } & \multicolumn{2}{c}{ Nanoparticles } \\
\cline { 2 - 5 } & Water & Ammonia & $\mathbf{A g}$ & $\mathbf{A l}_{\mathbf{2}} \mathbf{O}_{\mathbf{3}}$ \\
\hline$C_{P}$ & 4180 & 4740 & 235 & 765 \\
$\rho$ & 997 & 609 & 10500 & 3970 \\
$k$ & 0.607 & 0.521 & 429 & 40 \\
$\delta_{1}$ & & & 10.5 & 3.98 \\
$\delta_{2}$ & & & 0.59 & 0.729 \\
$\gamma$ & & & 714 & 65.25 \\
\hline
\end{tabular}

Table 2. Comparison of $R a_{c}^{s t}$ with various combinations ( $R i$, $\left.\phi_{v}\right)$

\begin{tabular}{cccc}
\hline & \multicolumn{3}{c}{$R a_{c}{ }^{s t}$} \\
\cline { 2 - 4 } & $\phi_{v}=0$ & $\phi_{v}=0.05$ & $\phi_{v}=0.1$ \\
\hline$R i=0$ & $1708.00[21]$ & $525.890[21]$ & $379.606[21]$ \\
\hline$R i=2$ & 1378.05 & 444.977 & 323.149 \\
\hline$R i=5$ & 958.598 & 329.978 & 241.719 \\
\hline$R i=10$ & 407.518 & 153.609 & 114.045 \\
\hline
\end{tabular}

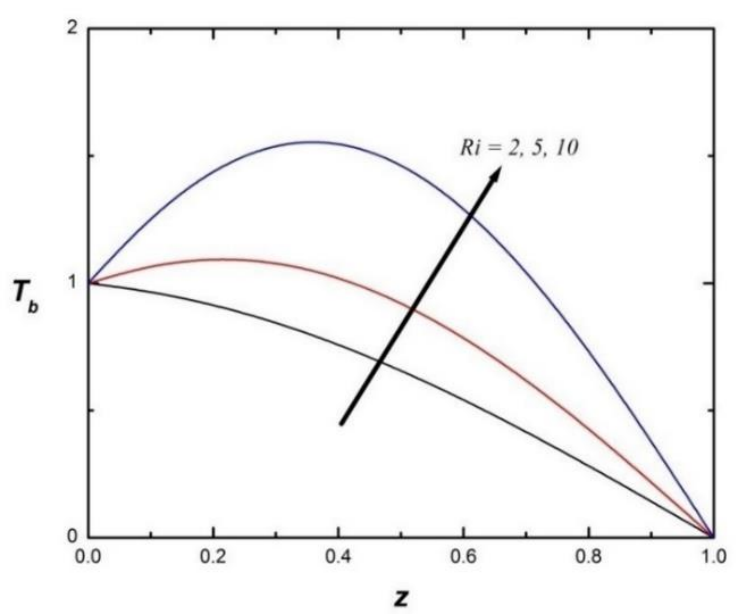

Figure 2. Basic temperature distribution $T_{b}$ vs. heat source parameter $R i$
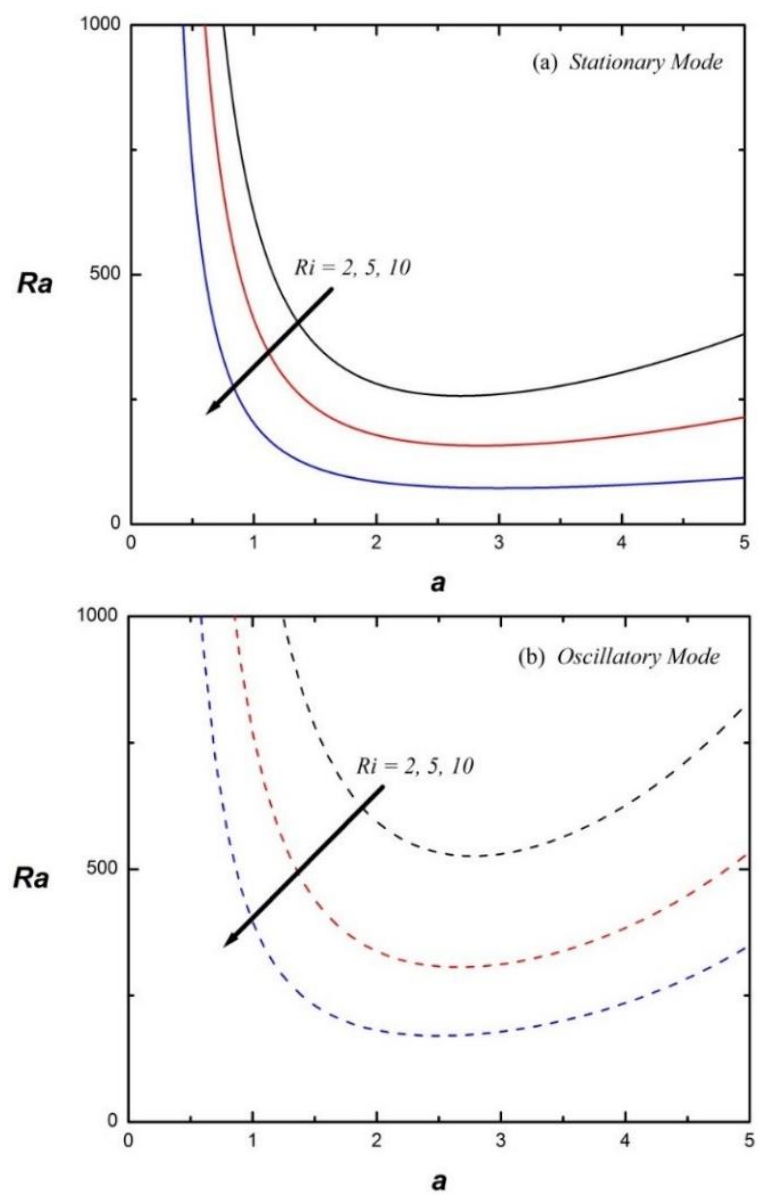

Figure 3. Thermal Rayleigh number $R a$ vs. wave number $a$ for distinct heat source parameter $R i$ 

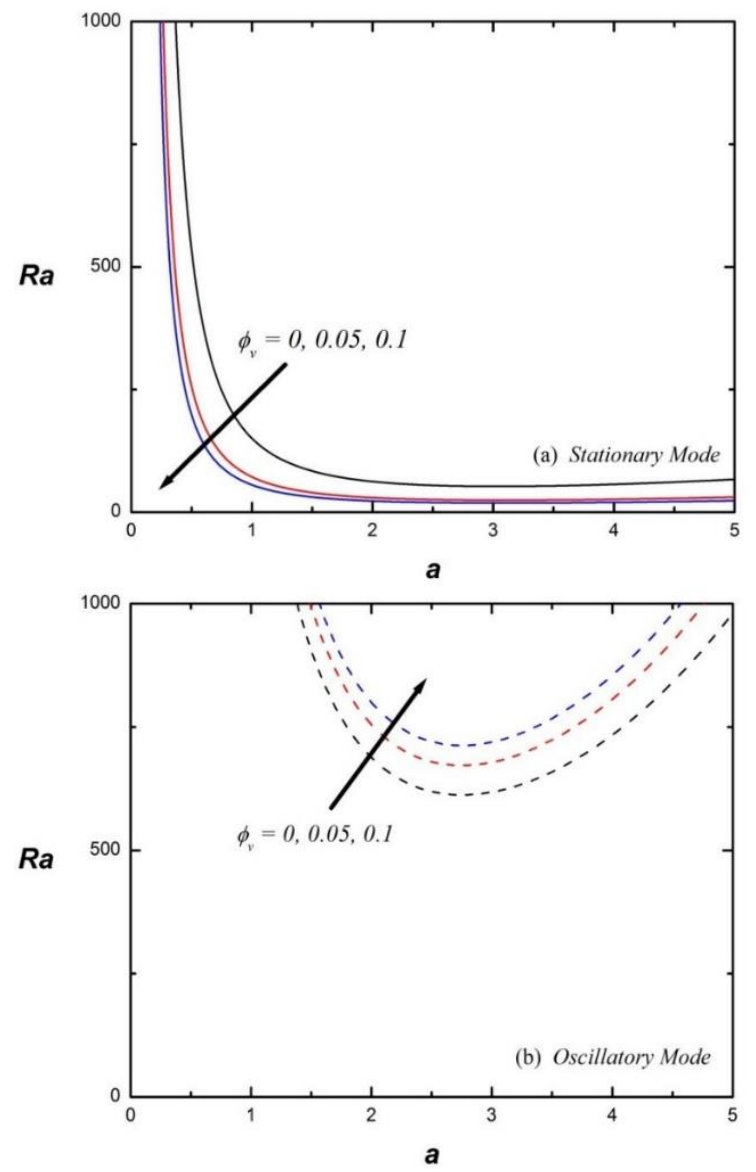

Figure 4. Thermal Rayleigh number $R a$ vs. wave number $a$ for distinct volume fraction of nanoparticles $\phi_{v}$
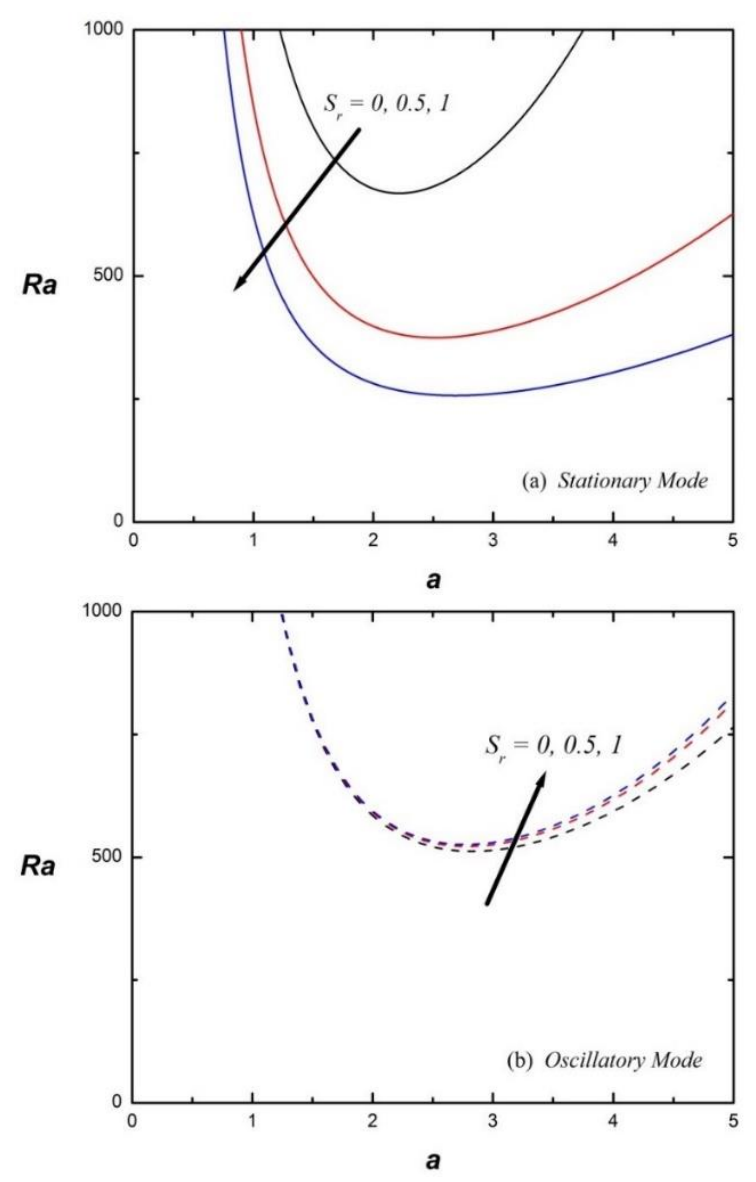

Figure 5. Thermal Rayleigh number $R a$ vs. wave number $a$ for distinct Soret parameter $S_{r}$
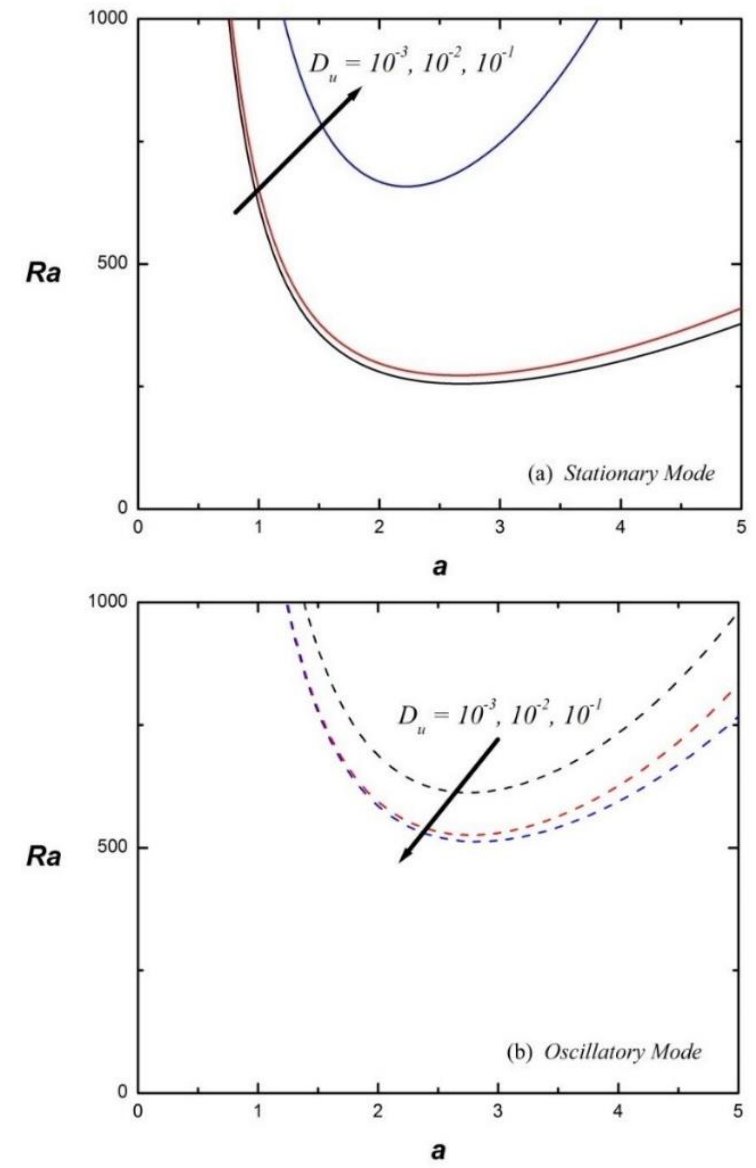

Figure 6. Thermal Rayleigh number $R a$ vs. wave number $a$ for distinct Dufour parameter $D_{u}$
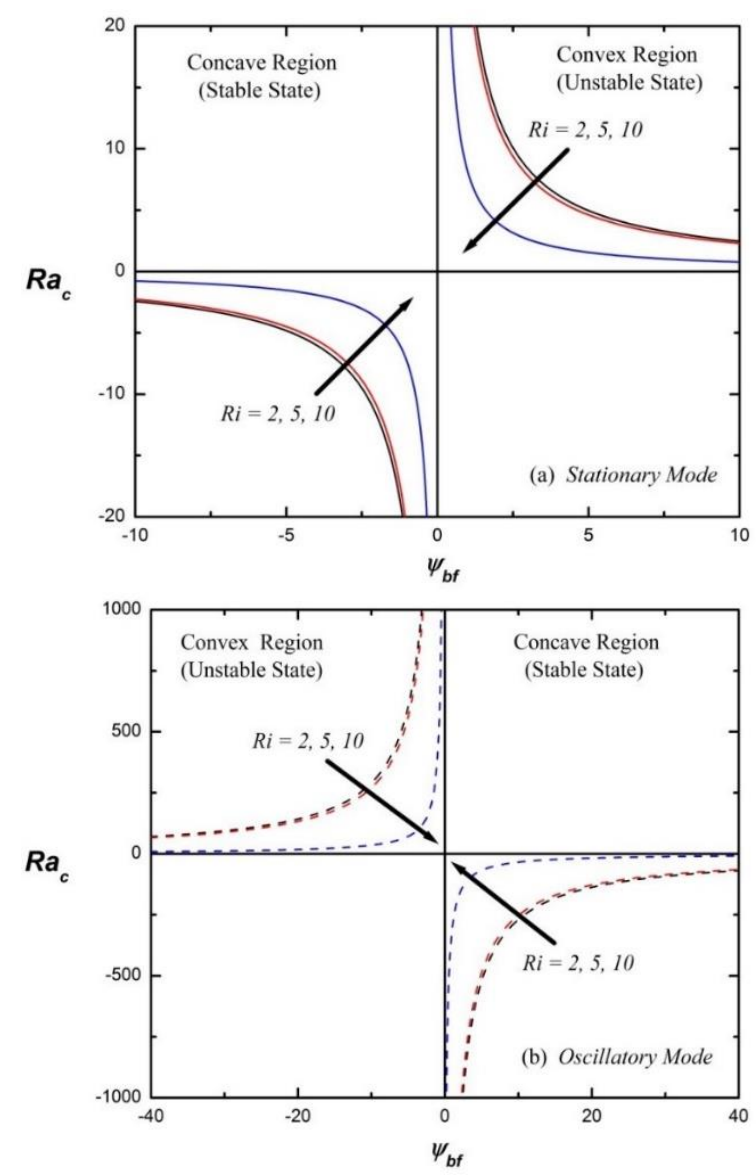

Figure 7. Heat source parameter $R i$ 's effect on space $\left(R a_{c}\right.$, $\left.\psi_{b f}\right)$ 

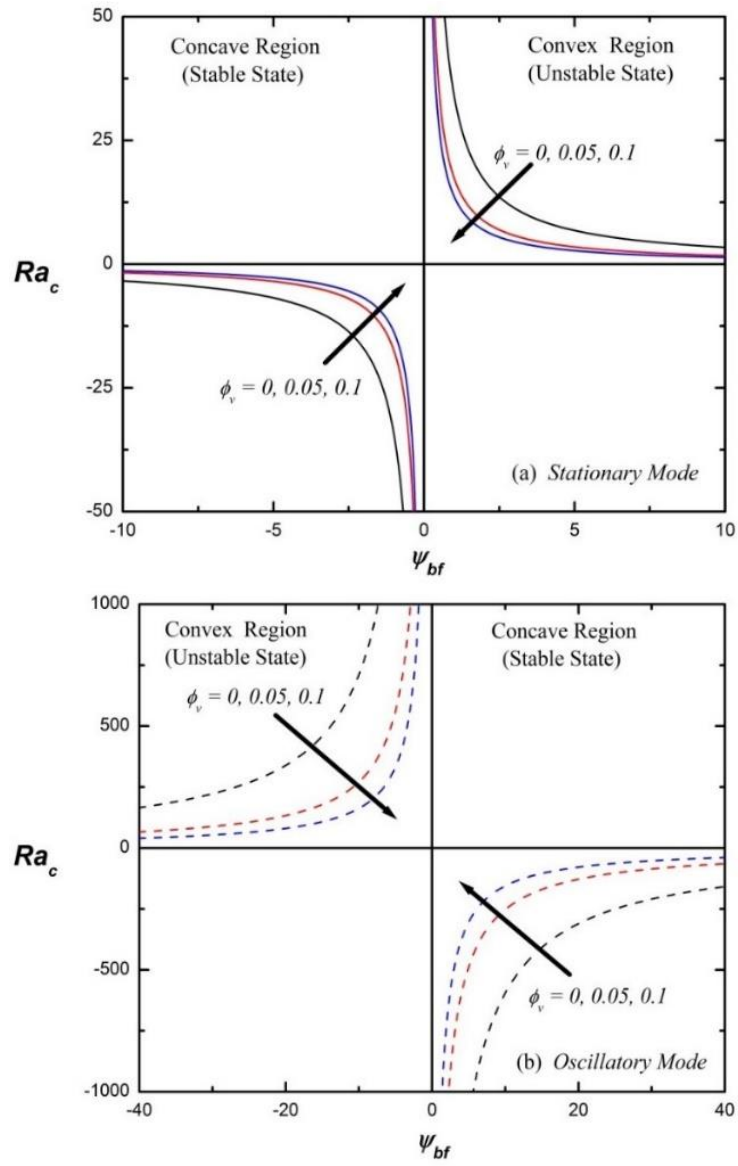

Figure 8. Volume fraction of nanoparticles $\phi_{v}$ 's effect on space $\left(R a_{c}, \psi_{b f}\right)$
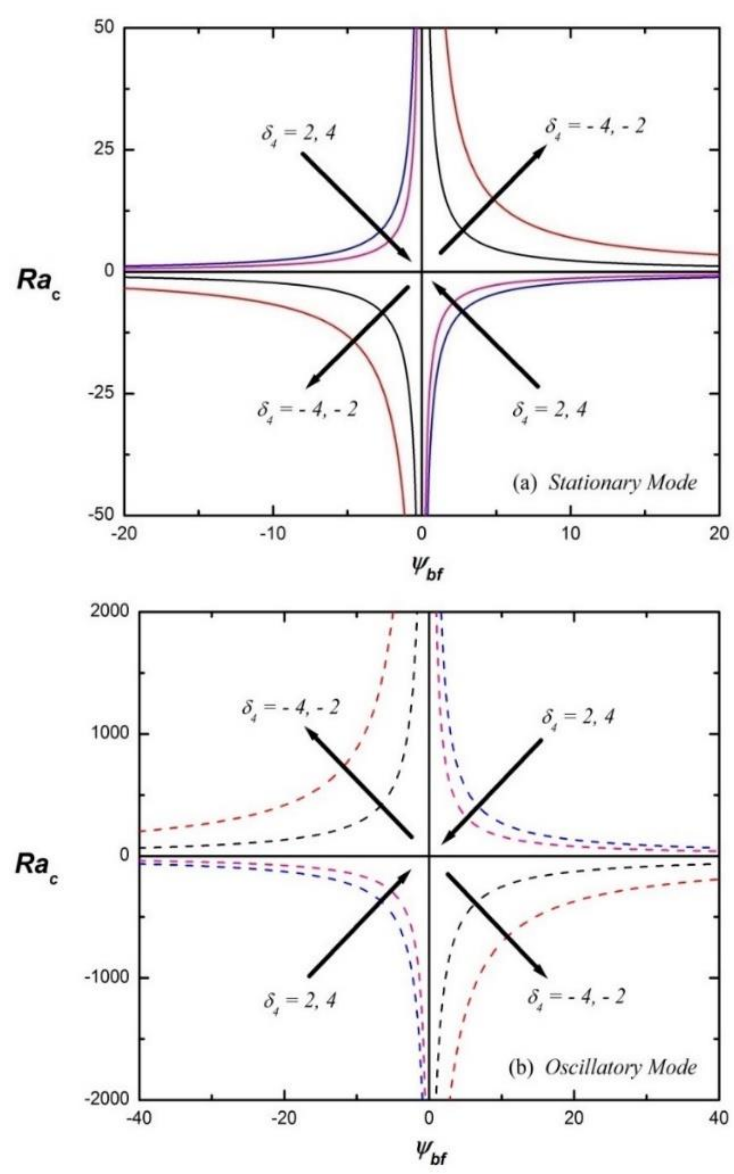

Figure 9. Soret effect of nanoparticles $\delta_{4}$ on space $\left(R a_{c}, \psi_{b f}\right)$
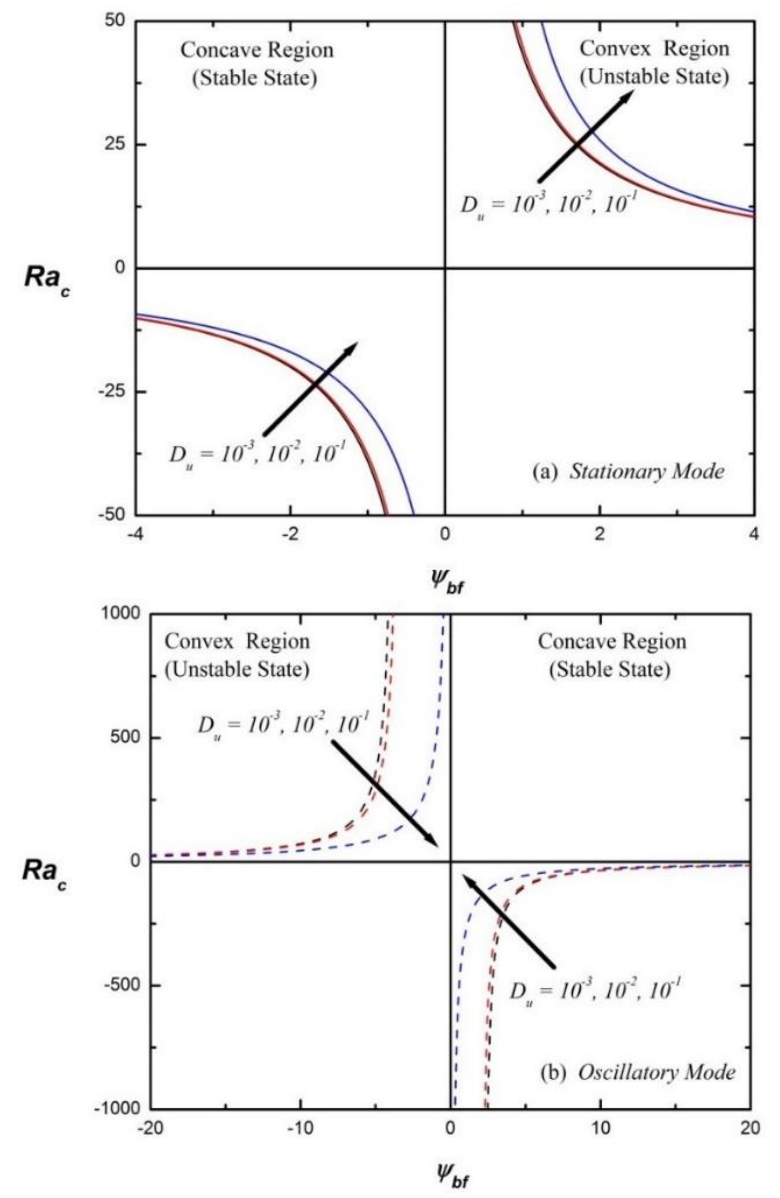

Figure 10. Dufour parameter $D_{u}$ 's effect on space $\left(R a_{c}, \psi_{b f}\right)$

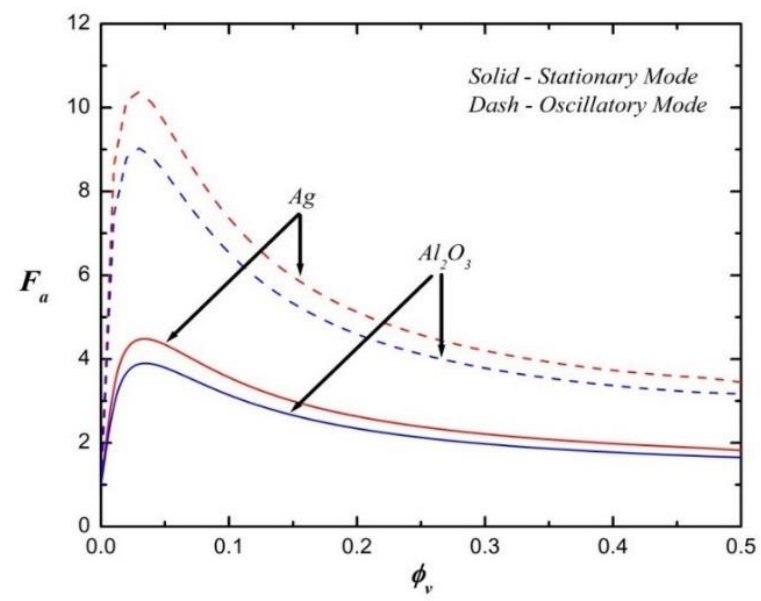

Figure 11. Addition factor $F_{a}$ vs. volume fraction of nanoparticles $\phi_{v}$

The convective stability is expressed through the stability curves. The concave and convex regions of the curves represent the stable and unstable states of the system respectively. The neutral stability curves are depicted in Figures $3-6$ for different values of heat source parameter $R i$, volume fraction of nanoparticles $\phi_{v}$, the Soret parameter $S_{r}$ and the Dufour parameter $D_{u}$ respectively. From the Figure 3 it is observed that as $R i$ increases, the thermal Rayleigh number $R a$ decreases in both stationary (Figure $3 \mathrm{a}$ ) and oscillatory (Figure $3 b)$ states. Thus, internal heating shrinks the convective cells and causes to evolve the onset of convective instability in the system. Further from the Figures 4, 5 and 6, it is found that the Rayleigh number $R a$ decreases with an increase of volume 
fraction $\phi_{v}$ and the Soret parameter $S_{r}$ and it increases with the Dufour parameter $D_{u}$ in stationary modes (Figures $4 \mathrm{a}, 5 \mathrm{a}, 6 \mathrm{a}$ ). Hence, $\phi_{v}$ and $S_{r}$ shrink the size of convection cells and $D_{u}$ enlarges the size of convection cells. If the size of convection cells is less, then the convective motion in the layer of binary nanofluid sets in easily and the layer is more unstable. Thus, volume fraction of nanoparticles $\phi_{v}$ and Soret parameter $S_{r}$ have destabilizing effect, and Dufour parameter $D_{u}$ has stabilizing effect on the system. The opposite trend is noticed in case of oscillatory modes (Figures $4 b, 5 b, 6 b$ ).

Figures 7 and 8 are the graphs of critical Rayleigh number $R a_{c}$ versus separation ratio in binary basefluid $\psi_{b f}$ for various internal heat source parameter $R i$ and volume fraction of nanoparticle $\phi_{v}$ respectively. In stationary mode (Figures 7a and 8a), it is observed that the critical Rayleigh number $R a_{c}$ decreases sharply with respect to absolute values of $\psi_{b f}$ but in oscillatory mode (Figures $7 \mathrm{~b}$ and $8 \mathrm{~b}$ ), it happens exactly reverse. It implies that the convective motion is produced easily with support of the separation ratio in binary basefluids i.e. Soret effect of solute $\psi_{b f}$. From the graphs, it has been realized that the stable state region is reduced and unstable state region is enlarged with an increase in the values of $R i$ and $\phi_{\nu}$ in both the modes of stationary and oscillatory convections. It signifies that the heat source parameter $R i$ and the volume fraction of nanoparticles $\phi_{v}$ have destabilizing effect on the system.

Soret effect of nanoparticles represented by the factor $\delta_{4}$ on the space $\left(R a_{c}, \psi_{b f}\right)$ is illustrated in Figure 9 . For $\delta_{4}>0$, It is detected that the $R a_{c}$ decreases with an increase of $\delta_{4}$ and increases with respect to the Soret effect of solute $\psi_{b f}$. Thus, the thermodiffusion of nanoparticles strengthen the Brownian motion whereas thermodiffusion of solute weaken the motion in mode of stationary state (Figure 9a). Furthermore, it has been observed that the $R a_{c}$ decreases with an increase of $\delta_{4}$ with respect to $\psi_{b f}$ in mode of oscillatory state. Here, thermodiffusion effects of both nanoparticles and solute strengthen the Brownian motion in binary nanofluid. The reverse effects have been noted for $\delta_{4}<0$. Through the above discussion, it is worked out that $\delta_{4}$ plays key role to decide the perfect pair of nanoparticle and solute for strengthening the convective heat transport in binary nanofluids in the presence of internal heat source.

Dufour effect represented by the factor $D_{u}$ on the Rayleigh number $R a_{c}$ with respect to separation ratio of basefluid $\psi_{b f}$ is shown in Figure 10. In stationary mode (Figure 10a), the effect of Dufour parameter $D_{u}$ is insignificant on the stability states of the system i.e. there is no variation in the stability of the system, whereas its impact is considerable in case of oscillatory mode (Figure 10b). The stable state region becomes smaller and unstable state region becomes wider. Thus, the Dufour parameter helps to rise the onset of oscillatory convection in the system.

The profiles of addition factor $F_{a}$ versus volume fraction $\phi_{v}$ are depicted in Figure 11. Due to lesser thermal conductivity of alumina than silver, the profile curves of addition factor $F_{a}$ are always lie below in case of alumina based binary nanofluid of those in case of silver based binary nanofluid. Moreover, it is noticed that, the profiles of addition factor $F_{a}$ increase up to particular value with an increase of volume fraction of nanoparticles then decrease gradually. It indicates that a little addition of nanoparticles in the basefluids causes to improve the transport of concentration and heat in binary nanofluids.

\section{CONCLUSIONS}

In the present work, investigation on the influence of internal heating with cross diffusions on convective stability of the system and heat transfer enhancement in binary nanofluids is made. From the above discussion and results, the following observations are summarized:

- The size of convection cells decreases with an increase in the values of internal Rayleigh number in both stationary and oscillatory states of the system.

- With an increase in the values of Dufour parameter, the size of convection cells increases and with volume fraction of nanoparticles and Soret coefficient, decreases in stationary mode. The effects are reversed in oscillatory mode.

- The internal heat source parameter i.e. internal Rayleigh number causes to increase the transfer of heat and concentration in the system.

- Stability of the system under the influence of cross diffusions depends upon the magnitudes of Soret effects of solute $\psi_{b f}$ and nanoparticles $\delta_{4}$.

- Due to decrease in the addition factor $F_{a}$ gradually in respect of volume fraction of nanoparticles $\phi_{v}$ causes to increase the heat transfer in binary nanofluid.

The results of the present study are useful to analyze the convective instability of binary nanofluids which are used as working fluids in many industrial applications such as absorption refrigeration and air conditioning, freezing of foods, solar collectors, medical treatment, electro and electroless plating, etc. They provide a good guideline to choose the best pair of nanoparticle and solute in binary nanofluids.

\section{ACKNOWLEDGEMENT}

The authors acknowledge the University Grant Commission (UGC), Government of India, New Delhi for financial support under the scheme 'Maulana Azad National Research Fellowship'. (F. No. 61-1/2019 (SA - III), dated 14/08/2019).

\section{REFERENCES}

[1] Das, S.K., Choi, S.U.S., Yu, W., Pradeep, T. (2007). Nanofluids: Science and Technology. John Wiley and Sons, Inc., Hoboken, New Jersey, pp. 337-352. https://doi.org/10.1002/9780470180693

[2] Wang, L., Quintard, M. (2009). Nanofluids of the Future. In: Wang L. (eds) Advances in Transport Phenomena. Advances in Transport Phenomena, vol 1. Springer, Berlin, Heidelberg. https://doi.org/10.1007/978-3-64202690-4_4

[3] Choi, S., Eastman, J.A. (1995). Enhancing Thermal Conductivity of Fluids with Nanoparticles. ASME Fluids Engineering Division, 231: 99-105.

[4] Ramanathan, A., Gunasekaran, P. (2008). Simulation of absorption refrigeration system for automobile application. Thermal Sciences, 12(3): 5-13. https://doi.org/10.2298/TSCI0803005R

[5] Kuznetsov, I.A., Greenfield, M.J., Mehta, Y.V., Merchan, W., Salkar, G., Saveliev, A.V. (2011). Increasing the solar cell power output by coating with transition metal oxide nanorods. Applied Energy, 88(11): 4218-4221. https://doi.org/10.1016/j.apenergy.2011.04.033 
[6] Singh, R.P., Heldman, D.R. (2014). Introduction to Food Engineering. 4th ed. Academic Press, San Diego, California, USA. https://doi.org/10.1016/C2011-006101-X

[7] Kim, J.K., Jung, J.Y., Kang, Y.T. (2007). Absorption performance enhancement by nanoparticles and chemical surfactants in binary nanofluids. International Journal of Refrigeration, 30(1): $50-57$ https://doi.org/10.1016/j.ijrefrig.2006.04.006

[8] Ma, X., Su, F., Chen, J., Zhang, Y. (2007). Heat and mass transfer enhancement of the bubble absorption for a binary nanofluid. Journal of Mechanical Science and Technology, 21:

$1813-1818$ https://doi.org/10.1007/BF03177437

[9] Lee, J.K., Koo, J., Hong, H., Kang, Y.T. (2010). The effect of nanoparticles on absorption heat and mass transfer performance in $\mathrm{NH}_{3} / \mathrm{H}_{2} \mathrm{O}$ binary nanofluid. International Journal of Refrigeration, 33(2): 269-275. https://doi.org/10.1016/j.ijrefrig.2009.10.004

[10] Pang, C., Wu, W., Sheng, W., Zhang, H., Kang, Y.T. (2012). Mass transfer enhancement by binary nanofluid $\left(\mathrm{NH}_{3} / \mathrm{H}_{2} \mathrm{O}+\mathrm{Ag}\right.$ Nanoparticles) for bubble absorption process. International Journal of Refrigeration, 35(8): 2240-2247.

http://dx.doi.org/10.1016/j.ijrefrig.2012.08.006

[11] Xuan, Y. (2009). Conception for enhanced mass transport in binary nanofluids. Heat and Mass Transfer, 46: 277-279. https://doi.org/10.1007/s00231-009-0564-z

[12] Chand, R., Rana, G.C. (2012). On the onset of thermal convection in rotating nanofluid layer saturated DarcyBrinkman model. International Journal of Heat and Mass Transfer, 55(21-22): 5417-5424 http://dx.doi.org/10.1016/j.ijheatmasstransfer.2012.04.0 43

[13] Chand, R., Rana, G.C. (2012). Thermal instability of Rivlin - Ericksen elastic-viscous nanofluid saturated by porous medium. Journal of Fluid Engineering, 134(12): 1-7. https://doi.org/10.1115/1.4007901

[14] Buongiorno, J. (2005). Convective transport in nanofluids. Journal of Heat and Mass Transfer, 128(3): 240-250. https://doi.org/10.1115/1.2150834

[15] Nield, D.A., Kuznetsov, A.V. (2010). The onset of double diffusive nanofluid convection in a layer of a saturated porous medium. Transport in Porous Media, 85: 941-951. https://doi.org/10.1007/s11242-010-9600-1

[16] Yadav, D., Agarwal, G.S., Bhargava, R. (2012). The onset of convection in a binary nanofluid saturated porous layer. International Journal of Theoretical and Applied Multiscale Mechanics, 2(3): 198-224. https://doi.org/10.1504/IJTAMM.2012.049931

[17] Bhadauria, B.S., Agarwal, S., Sachetil, N.C., Chandran, P., Singh, A.K. (2012). Nonlinear convective transport in a binary nanofluid saturated porous layer. Transport in Porous Media, 93: 29-49. https://doi.org/10.1007/s11242-012-9942-y

[18] Gupta, U., Sharma, J., Sharma, V. (2015). Instability of binary nanofluid with magnetic field. Applied Mathematics and Mechanics, 36(6): 693-706. https://doi.org/10.1007/s10483-015-1941-6

[19] Kim, J., Kang, Y.T., Choi, C.K. (2003). Analysis of convective instability and heat transfer characteristics of nanofluids. Physics of Fluids, 16: 2395-2401. https://doi.org/10.1063/1.1739247

[20] Kim, J., Kang, Y.T., Choi, C.K., Kim, M.G. (2005).
Effects of thermodiffusion and nanoparticles on convective instabilities in binary nanofluids. Journal of Nanoscale and Microscale Thermophysical Engineering, $10(1)$

29-39. https://doi.org/10.1080/10893950500357772

[21] Kim, J., Kang, Y.T., Choi, C.K. (2007). Soret and Dufour effects on convective instabilities in binary nanofluids for absorption application. International Journal of Refrigeration, 30 : https://doi.org/10.1016/j.ijrefrig.2006.04.005

[22] Gaikwad, S.N., Kouser, S. (2014). Double diffusive convection in couple stress fluid saturated porous layer with internal heat source. International Journal of Heat and Mass Transfer, 78: 1254-1264. https://doi.org/10.1016/j.ijheatmasstransfer.2014.07.021

[23] Yadav, D., Wang, J. (2018). Convective heat transport in a heat generating porous layer saturated by a NonNewtonian nanofluid. Heat Transfer Engineering, 40(16) 1363-1382. https://doi.org/10.1080/01457632.2018.1470298

[24] Yadav, D., Bhargava, R., Agrawal, G.S. (2012). Effect of internal heat source on the onset of convection in nanofluid layer. Applied Mechanics of Materials, 110116: $1827-1832$ https://doi.org/10.4028/www.scientific.net/AMM.110116.1827

[25] Nield, D.A., Kuznetsov, A.V. (2013). The onset of convection in an internally heated nanofluid layer. Journal of Heat Transfer, ASME Series, 136(1): 014501.

[26] Kanchana C., Zhao, Y. (2018). Effect of internal heat generation/absorption on Rayleigh - Benard convection in water well-dispersed with nanoparticles or carbon nanotubes. International Journal of Heat and Mass Transfer, 127: 1031-1047. https://doi.org/10.1016/j.ijheatmasstransfer.2018.06.12

[27] Gaikwad, S.N., Surwase, D.M. (2020). Convective instability in binary nanofluids with an internal heat source in the presence of thermodiffusion and nanoparticles. Heat Transfer, 49(8): 4511-4527. https://doi.org/10.1002/htj.21838

[28] Hort, W., Linz, S.J., Lucke, M. (1992). Onset of convection in binary gas mixtures: Role of the Dufour effect. Physics Review A, 45(6): 3737-3748. https://doi.org/10.1103/PhysRevA.45.3737

[29] Drazin, P.G., Reid, W.H. (1981). Hydrodynamics Stability. Cambridge University Press, London.

[30] Chandrasekhar, S. (1961). Hydrodynamic and Hydromagnetic Stability. Oxford University Press, London.

[31] Cengel, Y.A. (1998). Heat Transfer: A Practical Approach. WCB/McGraw Hill, New York.

[32] Razif, N.H.M., Mamata, A.M.I.B., Lias, I., Mohamed, W.A.N.W. (2015) Thermophysical properties analysis for ammonia - water mixture of an organic Rankine cycle. Journal Teknologi (Science and Engineering), 75(8): 1317. https://doi.org/10.11113/jt.v75.5203

\section{NOMENCLATURE}

\section{Latin symbols}




\section{Greek letters}

$\alpha$

$\beta_{S}$

$\beta_{T}$

$\mu$

$v$

$\rho$

$\phi_{v}$

$\psi$

$\omega$

\section{Subscripts}

$\begin{array}{ll}b & \text { basic state } \\ b f & \text { binary basefluid } \\ n f & \text { nanofluid } \\ n p & \text { nanoparticle }\end{array}$

$s \quad$ solute quantity depth of nanofluid layer, $\mathrm{m}$

solute diffusivity, $\mathrm{m}^{2} \cdot \mathrm{s}^{-1}$

diffusivity by Soret type

diffusivity by Dufour type

differential operator $(=d / d z)$

Dufour parameter

addition factor

gravitational acceleration, $\mathrm{m}^{2} \cdot \mathrm{s}^{-1}$

thermal conductivity, $\mathrm{W} \cdot \mathrm{m}^{-3} \cdot \mathrm{K}^{-1}$

Lewis number

pressure, $\mathrm{Pa}$

Prandtl number

amount of heat flux

thermal Rayleigh number

solute Rayleigh number

internal Rayleigh number

solutal parameter

Soret parameter

temperature, $\mathrm{K}$

velocity vector, $\mathrm{m} \cdot \mathrm{s}^{-1}$

effective volume of nanoparticles

vertical velocity

thermal diffusivity, $\mathrm{m}^{2} \cdot \mathrm{s}^{-1}$

solute expansion

thermal expansion

dynamic viscosity

kinetic viscosity

density, $\mathrm{kg} \cdot \mathrm{m}^{-3}$

volume fraction of nanoparticles

separation ratio $\left(D \beta_{s} / D_{s r} \beta_{T}\right)$

frequency of oscillations

nanoparticle

\section{Other symbols}

$$
\begin{aligned}
& \nabla^{2}=\frac{\partial^{2}}{\partial x^{2}}+\frac{\partial^{2}}{\partial y^{2}}+\frac{\partial^{2}}{\partial z^{2}} \\
& \nabla_{1}^{2}=\nabla^{2}-\frac{\partial^{2}}{\partial z^{2}}
\end{aligned}
$$

\section{APPENDIX}

$\Delta_{1}=\frac{A_{1} B_{6} \omega^{2}+A_{2} B_{7}}{B_{4} \omega^{2}+B_{5}}$

$\Delta_{2}=\frac{A_{2} B_{6}-A_{1} B_{7}}{B_{4} \omega^{2}+B_{5}}$

$A_{1}=2 F \delta^{2}\left(1+S_{r}\right)-\delta^{2} D_{u}-S_{o}\left(\delta^{2}+R i\right)$

$A_{2}=2 F L e-S_{o}$

$A_{3}=\delta^{2}(1-K)+R i$

$A_{4}=\delta^{2} L e+\frac{\delta^{2}}{\operatorname{Pr}}+\frac{\delta^{2} L e}{\operatorname{Pr}}+\frac{L e R i}{\operatorname{Pr}}$

$A_{5}=\operatorname{Pr}^{-1} L e$

$A_{6}=\delta^{2}+L e\left(\delta^{2}+R i\right)+\frac{A_{3}}{\operatorname{Pr}}$

$B_{1}=A_{1} A_{5}$

$B_{2}=A_{1} A_{6}-A_{2} A_{4}$

$B_{3}=A_{2} A_{3}$

$B_{4}=A_{1}^{2}$

$B_{5}=A_{2}^{2}$

$B_{6}=\delta^{2}\left(B_{1}-\omega^{2} L e\right)$

$B_{7}=\delta^{4} B_{6}-\omega^{2} B_{5}$ 